\title{
Antidandruff activity of Cassia auriculata and Cassia alata through fatty acids mediated inhibition of Malassezia furfur
}

\author{
V. Prabhu* \\ PG Department of Chemistry, Nallamuthu Gounder Mahalingam College, Pollachi (Tamil Nadu), \\ India \\ K. Poonkodi \\ PG Department of Chemistry, Nallamuthu Gounder Mahalingam College, Pollachi (Tamil Nadu), \\ India \\ K. Pradeep \\ PG Department of Chemistry, Nallamuthu Gounder Mahalingam College, Pollachi (Tamil Nadu), \\ India \\ S. Buvaneswari \\ PG Department of Chemistry, Nallamuthu Gounder Mahalingam College, Pollachi (Tamil Nadu), \\ India \\ R. Mini \\ PG Department of Chemistry, Nallamuthu Gounder Mahalingam College, Pollachi (Tamil Nadu), \\ India \\ K. Vimaladevi \\ PG Department of Chemistry, Nallamuthu Gounder Mahalingam College, Pollachi (Tamil Nadu), \\ India \\ M. Anusuya \\ PG Department of Chemistry, Nallamuthu Gounder Mahalingam College, Pollachi (Tamil Nadu), \\ India \\ Sibi G \\ Department of Biotechnology, Indian Academy Degree College-Autonomous, Bangalore \\ (Karnataka), India \\ *Corresponding Author. Email: prabhusamruth@gmail.com
}

\section{Article Info}

https://doi.org/10.31018/

jans.v12i4.2390

Received: October 4, 2020

Revised: November 15, 2020

Accepted: November 20, 2020

\section{How to Cite}

Prabhu V. et al. (2020). Antidandruff activity of Cassia auriculata and Cassia alata through fatty acids mediated inhibition of Malassezia furfur. Journal of Applied and Natural Science, 12(4):532 - 540. https://doi.org/10.31018/jans.v12i4.2390

\begin{abstract}
Susceptibility of Malassezia furfur to certain medium chain fatty acids shed light onto novel strategies to control dandruff. This study explored antidandruff activity of the fatty acids and other bioactive compounds from flowers of Cassia auriculata and Cassia alata. The idea was supplementing the growth medium with fatty acids which are inhibitory to Malassezia so that plantbased antidandruff formulations could be developed based on the results. Chloroform and ethanolic flower extracts were tested there in vitro efficacy against $M$. furfur and the potential antidandruff compounds were identified by gas chromatography-mass spectrophotometry (GC-MS). Minimum inhibitory concentrations were determined for both the extracts and $I_{50}$ values of 50 and $88 \mu \mathrm{M}$ for chloroform extract of $C$. auriculata and C. alata were recorded. For ethanol extract, $\mathrm{IC}_{50}$ values of 75 and $70 \mu \mathrm{M}$ were exhibited by $C$. auriculata and $C$. alata, respectively. Inhibition of $M$. furfur through fatty acids from Cassia is the first report, and it is possible to include specific fatty acids in the growth media to inhibit the growth of Malassezia which could be later served as lead molecules in antidandruff formulations. Further, the presence of citronellol, pinitol, anthracenedione and chrysine in Cassia flower extracts and their antidandruff activity reported in this study needed further research on those compounds to formulate effective treatment of Malassezia associated diseases.
\end{abstract}

Keywords: Anti-dandruff, Cassia alata, Cassia auriculata, Fatty acids, Malassezia furfur

\section{INTRODUCTION}

Scalp has thick terminal hair, large numbers of sweat glands and sebaceous glands. Sebum secreted in stratum corneum provides a nutrient-enriched environment for microbial growth. Malassezia species are found on mammalian skin (Findley et al., 2013, Gemmer et al., 2002; Sampaio et al., 2011; Takemoto et al., 2015) associated with several common skin disorders (Sugita et al., 2010). Malassezia is a lipophilic yeast that uses sebum lipids as a nutrient 
source and leaves unsaturated free acids to result in dryness of the stratum corneum that ultimately leads to dandruff (Ashbee and Evans, 2002). Dandruff is characterized by redness on the scalp with loosely attached oily flakes on the skin (Gupta et al., 2004). Ketoconazole, zinc pyrithione, selenium sulfide and salicylic acid-containing preparations are commonly used in antidandruff shampoo formulations, but high resistance among Malassezia sp, recurrent dandruff rates and toxicity limits their usage on the human scalp.

Cassia belongs to the family Caesalpiniaceae and many studies have reported the use of Cassia due to its pharmacological potential. Various parts of Cassia auriculata and Cassia alata were studied for their antimicrobial activity (Murugan et al., 2013; Timothy et al., 2012). Cassia species is also reported in treating skin diseases (Singhal and Kansara, 2012; Khare 2007; Duke, 2002; Maity et al., 2001; Atarzadeh et al., 2017). Antifungal activity against Malassezia furfur by $C$. auriculata (Kumar et al., 2008) and C. alata (Damodaran and Venkataraman, 1994) were reported earlier. However, the active ingredients present in the plants were not explored from the point of antidandruff activity. Further, Malassezia spp. are susceptible to certain medium chain fatty acids (Papavassilis et al., 1999; Mayser, 2015; Bhattacharyya et al., 2017). In light of this information, this study explored antidandruff activity of the fatty acids and their derivatives from flowers of $C$. auriculata and $C$. alata. The idea was supplementing the growth medium with fatty acids which are inhibitory to Malassezia so that plant based antidandruff formulations could be developed based on the results.

\section{MATERIALS AND METHODS}

Plant materials: Fresh flowers of $C$. auriculata and $C$. alata were collected from Pollachi $\left(10.669823^{\circ} \mathrm{N}\right.$, $\left.76.980639^{\circ} \mathrm{E}\right)$, Tamil Nadu, India. The flowers were identified and authenticated in the Department of Botany, Bangalore University. The flowers were washed and air-dried and powdered.

Preparation of flower extract: Flowers of $C$. auriculata and C. alata $(50 \mathrm{~g})$ was extracted successively with $500 \mathrm{ml}$ of chloroform and $500 \mathrm{ml}$ of ethanol by using a soxhlet extractor for 4-6 $\mathrm{h}$ at a temperature not exceeding the boiling point. The chloroform and ethanol extracts were collected after filtration and evaporated to dryness under reduced pressure using a Rota vapour. The dried extracts were used for further studies.

Test organism: Malassezia furfur MTCC 1374 was obtained from Microbial Type Culture Collection, Chandigarh for this study.

Anti-malassezial activity: The antimicrobial activity of the test organisms to the $C$. auriculata and $C$. alata plant extracts was screened by using the agar well diffusion method. An inoculum suspension $\left(1.2 \times 10^{3}\right.$ CFU/ml) was swabbed uniformly to solidified $20 \mathrm{ml}$ Sabouraud's Dextrose Agar (SDA) and was allowed to dry for $5 \mathrm{~min}$. Holes of $6 \mathrm{~mm}$ in diameter were made in the seeded agar using a sterile cork borer. Aliquot of $10 \mu \mathrm{l}$ from each plant extract $(10,20,40,80,160$ and $320 \mu \mathrm{M}$ ) was added into each well on the seeded medium and allowed to stand on the bench for $1 \mathrm{~h}$ for proper diffusion and thereafter incubated at $37^{\circ} \mathrm{C}$ for $24 \mathrm{~h}$. The resulting inhibition zones were measured in millimetres $(\mathrm{mm})$. Ketoconazole was used as positive control and the studies were performed in triplicates.

Minimum inhibitory concentration assay: In vitro antidandruff activity was performed in 96 well microtitre plates using $M$. furfur MTCC 1374 in Sabouraud dextrose broth (Sibi et al., 2014). Extracts in the concentrations of $200 \mu \mathrm{M}, 180 \mu \mathrm{M}, 160 \mu \mathrm{M}, 140 \mu \mathrm{M}, 120$ $\mu \mathrm{M}, 100 \mu \mathrm{M}, 50 \mu \mathrm{M}, 25 \mu \mathrm{M}, 10 \mu \mathrm{M}$ and $1 \mu \mathrm{M}$ were tested against $M$. furfur. Cell density at $1.2 \times 10^{3} \mathrm{CFU} /$ $\mathrm{ml}$ was measured by following McFarland 0.5 standard solutions used. Optical density absorbance at $630 \mathrm{~nm}$ was read out in microtiter plate reader. Ketoconazole was used as a control in the study. The lowest concentration which inhibited the growth of the fungi was considered the minimum inhibitory concentration (MIC) of each extract. The inhibitory concentration $\left(I_{50}\right)$ value was calculated using the linear relation between the minimum inhibitory percentage and concentration of the extract.

GC-MS Profiling of bioactive compounds:To identify the compounds present in C. auiculata and C. alata, GC-MS analysis was carried out using an Agilent technologies GC systems with GC-7890A/MS-5975C model (Agilent Technologies, Santa Clara, CA, USA) equipped with HP-5MS column (30 m in length $\times 250$ $\mu \mathrm{m}$ in diameter $\times 0.25 \mu \mathrm{m}$ thickness of film). Spectroscopic detection by GC-MS involved an electron ionization system which utilized high energy electrons (70ev). Pure helium gas $(99.995 \%)$ was used as the carrier gas with a flow rate of $1 \mathrm{ml} / \mathrm{min}$. The initial temperature was set at $45-150^{\circ} \mathrm{C}$ with the increasing rate of $8^{\circ} \mathrm{C} / \mathrm{min}$ and holding time of about $4 \mathrm{~min}$. Finally, the temperature was increased to $290^{\circ} \mathrm{C}$ for $4 \mathrm{~min}$. One microliter of the prepared $1 \%$ of the extracts diluted with respective solvents was injected in a splitless mode. The relative quantity of the chemical compounds presents in each of the extracts of $C$. auriculata and $C$. alata was expressed as a percentage based on peak area produced in the chromatogram. Compounds were identified by comparing the mass spectral data with the NIST library (Prabhu et al., 2020).

Statistical analysis: The experiments were done in triplicate and the data reported as the mean \pm SD. Analysis of variance was performed by one way ANOVA using SPSS statistical software, version 20. A probability value at $P<0.05$ was considered statistically significant.

\section{RESULTS AND DISCUSSION}

An antidandruff activity using well diffusion assay revealed the inhibitory activity of both $C$. auriculata and $C$. alata against $M$. furfur. were prepared in chloroform 
and ethanol. Quantitative results of the effect of chloroform and ethanol extracts of flowers at the concentrations of $20,40,60,80$ and $100 \mu \mathrm{g} / \mathrm{ml}$ on the size of the clear zones are represented in the table-1. The range of inhibition values observed between the extracts varied, and not all preparations exhibited the same anti-malassezzial properties.

Anti-malassezial activity of ethanol extracts was higher than chloroform extract and the inhibitory was increased when the extract concentrations were higher. It was found that inhibitory concentration $\left(\mathrm{IC}_{50}\right)$ values of 50 and $88 \mu \mathrm{M}$ were recorded for chloroform extract of $C$. auriculata and C. alata flowers, respectively. For ethanol extract, $I_{50}$ values of 75 and $70 \mu \mathrm{M}$ were exhibited by $C$. auriculata and $C$. alata respectively (Fig. 1).

The peaks are marked with retention time in the GCMS chromatogram of the chloroform and ethanol extract of the flowers of Cassia auriculata and Cassia alata. Their retention time (RT) and the amount of their presence are indicated in Table 2, 3, 4, 5. A total of 59 and 37 compounds identified in chloroform and ethanol extract of $C$. auriculata and $C$. alata are shown in Fig. 2 and 3.

GC-MS analysis of chloroform extracts indicated the presence of fatty acids (i-Propyl 11,12-methyleneoctadecanoate, Octadecanoic acid, Hexadecanoic acid), fatty alcohols (Tetracosanol, Hexadecanol), sesquiterpenes and aromatic organic compounds in $C$. auriculata and $C$. alata. Of the total 59 compounds identified in the chloroform extracts, 30 were present in $C$. auriculata. The various compounds observed from chloroform extracts of $C$. auriculata and $C$. alata did not differ significantly from each other.

Fatty acids are carboxylic present in various plants as either saturated or unsaturated compounds with known antimicrobial activity (Rahuman et al., 2000; Cerdeiras et al., 2000; Dilika et al., 2000; Lee et al., 2002; McGraw et al., 2002; Kumar et al., 2010). Similarly, fatty acids esterified into polyhydric alcohols with antimicrobial effectiveness is reported (Mukherjee et al., 2013; Togashi et al., 2007; Kubo et al., 1993). In our study, anti-malassezial activity of chloroform extracts of Cassia was due to the presence of fatty acids and fatty alcohols. This is the novel report that fatty

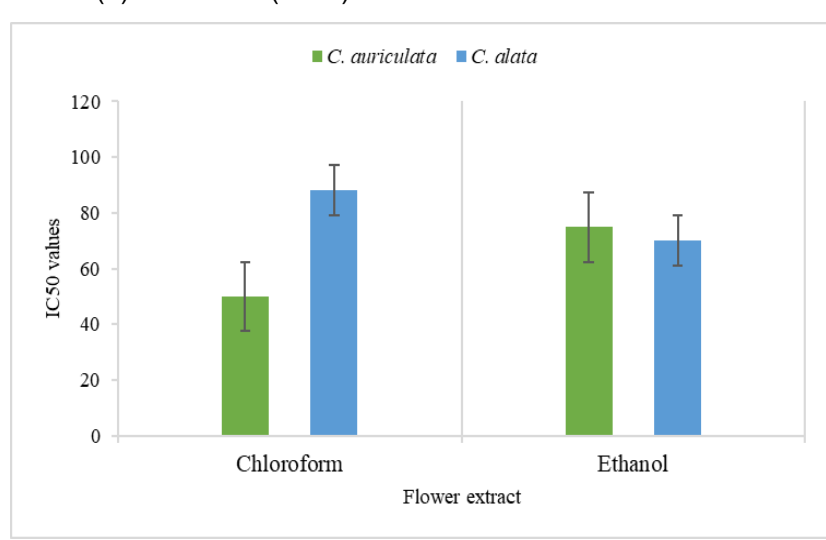

Fig. 1. $I C_{50}$ values of $C$. auriculata and $C$. alata flower extracts against $M$. furfur MTCC 1374.

acids from Cassia is inhibitory to $M$. furfur thus could help in the control of dandruff.

Anti-malassezial activity of medium-chain fatty acids is reported in earlier studies (Mayser, 2015; Bhattacharyya et al., 2017), but the presence of fatty acids and fatty alcohols in Cassia species and their inhibitory activity against Malassezia furfur is reported for the first time through this study. From the results, it is possible to include specific fatty acids in the growth media to inhibit the growth of Malassezia, which could be later served as lead molecules in antidandruff formulations.

Various bioactive compounds were present in ethanol extract of $C$. auriculata and $C$. alata as revealed from GC-MS analysis. Some of the important phytoconstituents identified in this study were terpenoids (Citronellol), polyols (pinitol), glycosides (Anthracenedione, Mannopyranoside) flavonoids (Resorcinol), flavones (Chrysine) along with carbohydrates (Fig. 4 and 5; Table 3 and 4). Antimicrobial activities of the above identified phytochemical compounds from various sources are widely documented in earlier studies. For example, resorcinol is known for its keratolytic and anti-dermatophytic activities (Romagnoli et al., 2016; Martindale, 1989). Ethanol extract of $C$. auriculata flowers showed the presence of resorcinol $(0.66 \%)$ in this study which may be attributed to the antidandruff activity of plant. Inhibitory activity of citronellol against the fungi Trichophyton

Table 1. Anti-malassezial activity of C.auriculata and C. alata flowers against M. furfur. (Values are of triplicates).

\begin{tabular}{lllll}
\hline \multirow{2}{*}{ Concentration $(\boldsymbol{\mu g} / \mathrm{ml})$} & \multicolumn{2}{c}{ Chloroform extract } & \multicolumn{2}{c}{ Ethanol extract } \\
\cline { 2 - 5 } & \multicolumn{2}{c}{ Zone of Inhibition $(\mathbf{m m})$} & \multicolumn{2}{c}{ Zone of Inhibition $(\mathbf{m m})$} \\
\cline { 2 - 5 } & C. auriculata & C. alata & C. auriculata & C. alata \\
\hline 20 & $0.0 \pm 0.0$ & $0.0 \pm 0.0$ & $0.0 \pm 0.00$ & $0.0 \pm 0.0$ \\
40 & $0.7 \pm 0.47$ & $0.3 \pm 0.06$ & $0.0 \pm 0.06$ & $0.1 \pm 0.01$ \\
80 & $3.3 \pm 0.11$ & $2.6 \pm 0.10$ & $3.1 \pm 0.20$ & $4.3 \pm 0.22$ \\
320 & $6.7 \pm 0.20$ & $4.7 \pm 0.01$ & $7.3 \pm 0.01$ & $7.7 \pm 0.03$ \\
Ketoconazole $(30 \mu \mathrm{g})$ & $6.8 \pm 0.25$ & $5.2 \pm 0.51$ & $7.1 \pm 0.33$ & $7.8 \pm 0.05$ \\
\hline
\end{tabular}


Table 2. Chemical constituents present in chloroform extract of $C$. auriculata flowers.

\begin{tabular}{|c|c|c|c|}
\hline Name of the compound & $\begin{array}{l}\text { Molecular } \\
\text { formula }\end{array}$ & $\begin{array}{l}\text { Retention time } \\
\text { (min) }\end{array}$ & Total Percentage \\
\hline Hexadecane & $\mathrm{C}_{16} \mathrm{H}_{34}$ & 14.927 & 0.583 \\
\hline 1-Octadecanol & $\mathrm{C}_{18} \mathrm{H}_{38} \mathrm{O}$ & 15.619 & 3.2 \\
\hline Heptadecane & $\mathrm{C}_{17} \mathrm{H}_{36}$ & 16.993 & 0.984 \\
\hline Pentadecane,2,6,10,14-tetramethyl- & $\mathrm{C}_{19} \mathrm{H}_{40}$ & 17.121 & 1.103 \\
\hline Octadecane & $\mathrm{C}_{18} \mathrm{H}_{38}$ & 18.968 & 1.910 \\
\hline Nonadecane & $\mathrm{C}_{19} \mathrm{H}_{40}$ & 20.883 & 1.455 \\
\hline Eicosane & $\mathrm{C}_{20} \mathrm{H}_{42}$ & 22.707 & 1.363 \\
\hline 1-Eicosene & $\mathrm{C}_{20} \mathrm{H}_{40}$ & 23.350 & 2.330 \\
\hline Heneicosane & $\mathrm{C}_{21} \mathrm{H}_{44}$ & 24.468 & 0.959 \\
\hline Docosane & $\mathrm{C}_{22} \mathrm{H}_{46}$ & 26.146 & 0.644 \\
\hline 1-Docosene & $\mathrm{C}_{22} \mathrm{H}_{44}$ & 26.778 & 2.054 \\
\hline Phenol,2,4-bis(1,1-dimethylethyl)- & $\mathrm{C}_{14} \mathrm{H}_{22} \mathrm{O}$ & 27.263 & 3.860 \\
\hline Tricosane & $\mathrm{C}_{23} \mathrm{H}_{48}$ & 27.775 & 0.996 \\
\hline Hentriacontane & $\mathrm{C}_{31} \mathrm{H}_{64}$ & 29.328 & 0.414 \\
\hline Hexadecanoic acid,butyl ester & $\mathrm{C}_{20} \mathrm{H}_{40} \mathrm{O}_{2}$ & 29.512 & 0.626 \\
\hline n-Tetracosanol-1 & $\mathrm{C}_{24} \mathrm{H}_{50} \mathrm{O}$ & 29.949 & 1.792 \\
\hline Pentacosane & $\mathrm{C}_{25} \mathrm{H}_{52}$ & 30.793 & 0.852 \\
\hline Phthalic acid, hept-4-yl isobutyl ester & $\mathrm{C}_{19} \mathrm{H}_{28} \mathrm{O}_{4}$ & 31.274 & 4.034 \\
\hline Phthalic acid, butyl-2-pentyl ester & $\mathrm{C}_{17} \mathrm{H}_{24} \mathrm{O}_{4}$ & 31.814 & 5.364 \\
\hline Heptacosane & $\mathrm{C}_{27} \mathrm{H}_{56}$ & 31.971 & 1.348 \\
\hline Dibutyl phthalate & $\mathrm{C}_{16} \mathrm{H}_{22} \mathrm{O}_{4}$ & 32.164 & 17.63 \\
\hline 1-Hexacosene & $\mathrm{C}_{26} \mathrm{H}_{52}$ & 32.399 & 3.072 \\
\hline $\begin{array}{l}\text { 12-Oxotricyclo [5.3.1.1(2,6)]dodeca-3,8-diene,11- } \\
\text { acetoxy-4,5,9-trichloro }\end{array}$ & $\mathrm{C}_{14} \mathrm{H}_{13} \mathrm{Cl}_{3} \mathrm{O}_{3}$ & 33.697 & 0.793 \\
\hline Nonacosane & $\mathrm{C}_{29} \mathrm{H}_{60}$ & 33.849 & 9.685 \\
\hline 17-Pentatriacontene & $\mathrm{C}_{35} \mathrm{H}_{70}$ & 34.196 & 1.247 \\
\hline I-(+)-Ascorbic acid 2,6-dihexadecanoate & $\mathrm{C}_{38} \mathrm{H}_{68} \mathrm{O}_{8}$ & 34.407 & 0.888 \\
\hline i-Propyl 11,12-methylene-octadecanoate & $\mathrm{C}_{22} \mathrm{H}_{42} \mathrm{O}_{2}$ & 35.145 & 1.768 \\
\hline Hexadecanoic acid & $\mathrm{C}_{18} \mathrm{H}_{36} \mathrm{O}_{2}$ & 36.103 & 0.632 \\
\hline Bis(2-ethylhexyl)phthalate & $\mathrm{C}_{24} \mathrm{H}_{38} \mathrm{O}_{4}$ & 37.089 & 26.104 \\
\hline Octadecane,-3-ethyl-5-(2-ethylbutyl)- & $\mathrm{C}_{26} \mathrm{H}_{54}$ & 37.289 & 2.33 \\
\hline
\end{tabular}

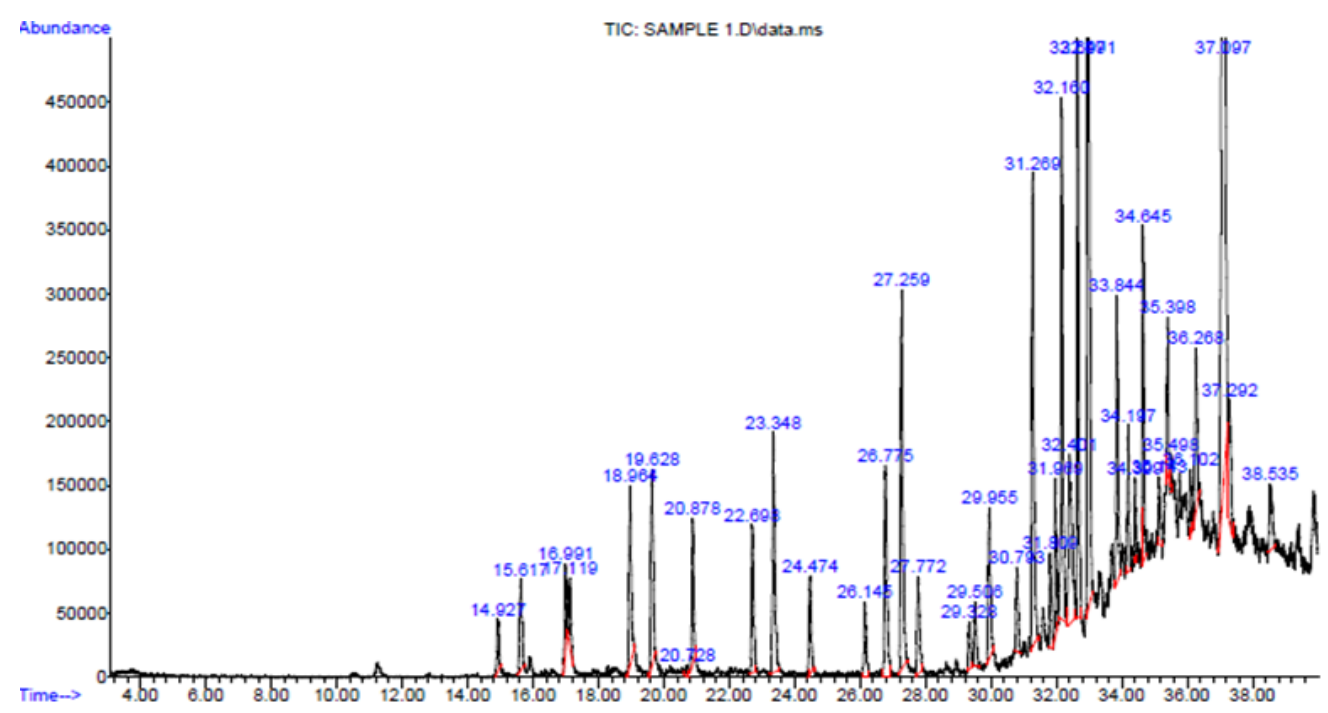

Fig. 2. Chromatogram of chloroform extract of $C$. auriculata flowers. 
Prabhu V. et al. / J. Appl. \& Nat. Sci. 12(4): 532 - 540 (2020)

Table 3. Chemical constituents present in chloroform extract of $C$. alata flowers.

\begin{tabular}{|c|c|c|c|}
\hline Name of the compound & $\begin{array}{l}\text { Molecular } \\
\text { Formula }\end{array}$ & $\begin{array}{l}\text { Retention time } \\
\text { (min) }\end{array}$ & $\begin{array}{l}\text { Total } \\
\text { Percentage }\end{array}$ \\
\hline Hexadecane & $\mathrm{C}_{16} \mathrm{H}_{34}$ & 15.012 & 0.556 \\
\hline 1-Hexadecanol & $\mathrm{C}_{16} \mathrm{H}_{34} \mathrm{O}$ & 15.704 & 0.975 \\
\hline Heptadecane & $\mathrm{C}_{17} \mathrm{H}_{36}$ & 17.031 & 0.875 \\
\hline Hexadecane, 2,6,10-trimethyl- & $\mathrm{C}_{19} \mathrm{H}_{40}$ & 17.134 & 1.015 \\
\hline Octadecane & $\mathrm{C}_{18} \mathrm{H}_{38}$ & 18.977 & 1.656 \\
\hline 1-Nonadecane & $\mathrm{C}_{19} \mathrm{H}_{38}$ & 19.635 & 0.752 \\
\hline Benzyl alcohol & $\mathrm{C}_{7} \mathrm{H}_{8} \mathrm{O}$ & 20.024 & 0.923 \\
\hline Nonadecane & $\mathrm{C}_{19} \mathrm{H}_{40}$ & 20.865 & 2.178 \\
\hline Eicosane & $\mathrm{C}_{20} \mathrm{H}_{42}$ & 22.673 & 1.757 \\
\hline 1-Eicosene & $\mathrm{C}_{20} \mathrm{H}_{40}$ & 23.308 & 1.006 \\
\hline Heneicosane & $\mathrm{C}_{12} \mathrm{H}_{44}$ & 24.413 & 1.856 \\
\hline Docosane & $\mathrm{C}_{22} \mathrm{H}_{46}$ & 26.112 & 1.683 \\
\hline Benzene,1,2,3-trimethoxy-5-(2-propenyl)- & $\mathrm{C}_{12} \mathrm{H}_{16} \mathrm{O}_{3}$ & 26.490 & 0.513 \\
\hline $\mathrm{n}$-Tetracosanol-1 & $\mathrm{C}_{24} \mathrm{H}_{50} \mathrm{O}$ & 26.713 & 0.788 \\
\hline 1,3-Benzodioxole, 4-methyl-6-(2-propyl)- & $\mathrm{C}_{11} \mathrm{H}_{12} \mathrm{O}_{3}$ & 26.879 & 0.265 \\
\hline Phenol, 2,4-bis(1,1-dimethylethyl)- & $\mathrm{C}_{14} \mathrm{H}_{22} \mathrm{O}$ & 27.154 & 3.196 \\
\hline Tetracosane & $\mathrm{C}_{24} \mathrm{H}_{50}$ & 27.726 & 6.097 \\
\hline Hexadecanoic acid, butyl ester & $\mathrm{C}_{20} \mathrm{H}_{40} \mathrm{O}_{2}$ & 29.437 & 0.279 \\
\hline Heptafluorobutyric acid, n-octadecyl ester & $\mathrm{C}_{22} \mathrm{H}_{37} \mathrm{~F}_{7} \mathrm{O}_{2}$ & 29.900 & 0.783 \\
\hline Pentacosane & $\mathrm{C}_{25} \mathrm{H}_{52}$ & 30.787 & 6.624 \\
\hline Phthalic acid, butyl isohexyl ester & $\mathrm{C}_{18} \mathrm{H}_{26} \mathrm{O}_{4}$ & 31.182 & 4.17 \\
\hline Tetratriacontane & $\mathrm{C}_{34} \mathrm{H}_{70}$ & 31.937 & 18.98 \\
\hline Phthalic acid, butyl hex-3-yl ester & $\mathrm{C}_{18} \mathrm{H}_{26} \mathrm{O}_{4}$ & 32.572 & 2.163 \\
\hline 1-(+)-Ascorbic acid 2,6-dihexadecanoate & $\mathrm{C}_{38} \mathrm{H}_{68} \mathrm{O}_{8}$ & 34.323 & 6.188 \\
\hline Nonacosane & $\mathrm{C}_{29} \mathrm{H}_{60}$ & 34.638 & 22.356 \\
\hline Octadecanoic acid & $\mathrm{C}_{18} \mathrm{H}_{36} \mathrm{O}_{2}$ & 36.000 & 1.890 \\
\hline Octadecane, 3-ethyl-5-(2-ethylbutyl)- & $\mathrm{C}_{26} \mathrm{H}_{54}$ & 37.281 & 5.694 \\
\hline (2S,2'S)-2,2'-Bis[1,4,7,10,13-pentaoxacyclopentadecane] & $\mathrm{C}_{20} \mathrm{H}_{38} \mathrm{O}_{10}$ & 37.922 & 1.702 \\
\hline 2-Hexadecanol & $\mathrm{C}_{16} \mathrm{H}_{34} \mathrm{O}$ & 41.361 & 5.080 \\
\hline
\end{tabular}

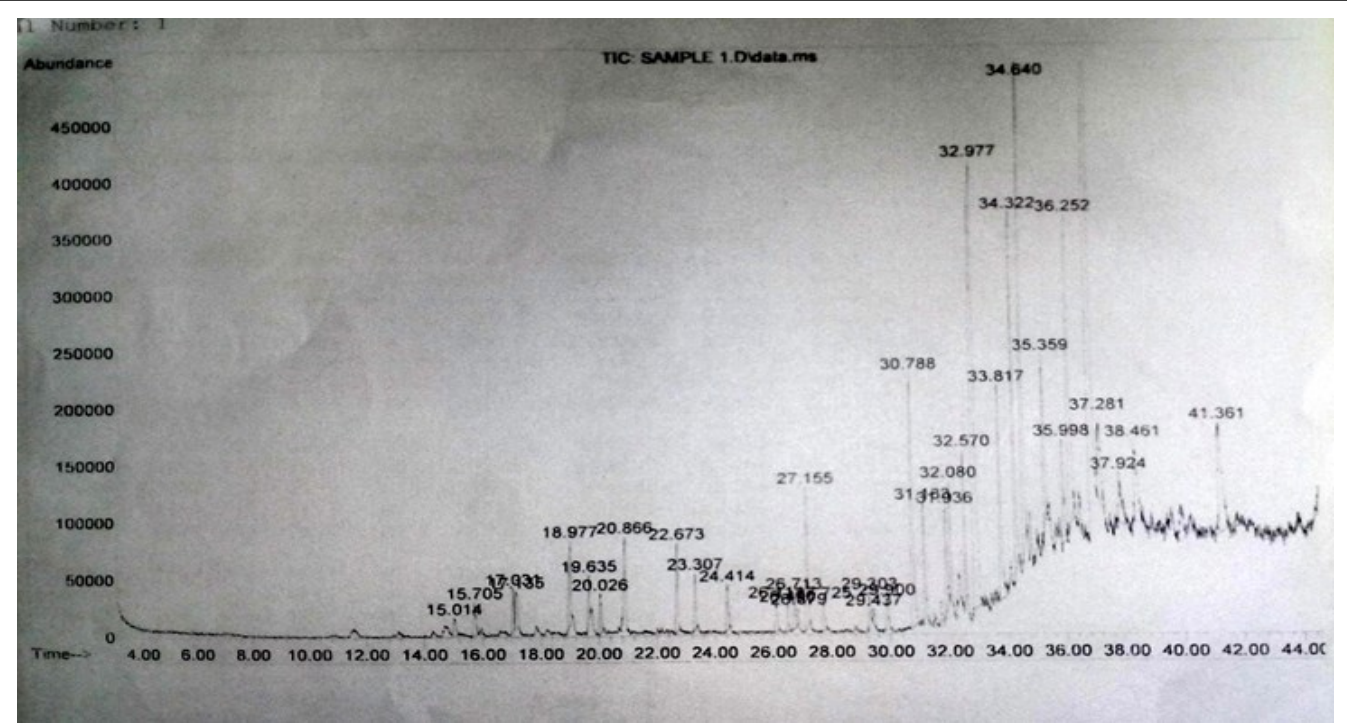

Fig. 3. Chromatogram of chloroform extract of C. alata flowers. 
Table 4. Chemical constituents present in ethanol extract of $C$. auriculata flowers.

\begin{tabular}{|c|c|c|c|}
\hline $\begin{array}{l}\text { Name of the } \\
\text { Compound }\end{array}$ & $\begin{array}{l}\text { Molecular } \\
\text { formula }\end{array}$ & $\begin{array}{l}\text { Retention } \\
\text { time }\end{array}$ & $\begin{array}{l}\text { Total } \\
\text { Percentage }\end{array}$ \\
\hline Resorcinol & $\mathrm{C}_{6} \mathrm{H}_{6} \mathrm{O}_{2}$ & 14.4722 & 0.665 \\
\hline 2-Oxiranemethanol, a-(1-methylethyl)-3-[1-(trimethylsilyloxy)pentyl]- & $\mathrm{C}_{14} \mathrm{H}_{30} \mathrm{O}_{3} \mathrm{Si}$ & 20.993 & 1.063 \\
\hline D-Pinitol, pentakis(trimethylsilyl) ether & $\mathrm{C}_{22} \mathrm{H}_{54} \mathrm{O}_{6} \mathrm{Si}_{5}$ & 21.713 & 3.600 \\
\hline 1,5-Anhydro-D-sorbitol, tetrakis(trimethylsilyl) ether & $\mathrm{C}_{18} \mathrm{H}_{44} \mathrm{O}_{5} \mathrm{Si}_{4}$ & 22.003 & 5.520 \\
\hline Lactulose, octakis(trimethylsilyl) ether (isomer 1) & $\mathrm{C}_{36} \mathrm{H}_{86} \mathrm{O}_{11} \mathrm{Si}_{8}$ & 22.324 & 5.002 \\
\hline Myo-Inositol, 1,2,3,4,5,6-hexakis-O-(trimethylsilyl)- & $\mathrm{C}_{24} \mathrm{H}_{60} \mathrm{O}_{6} \mathrm{Si}_{6}$ & 22.744 & 22.725 \\
\hline D-(-)- Erythrofuranose, tris(trimethylsilyl) ether (isomer 2) & $\mathrm{C}_{13} \mathrm{H}_{32} \mathrm{O}_{4} \mathrm{Si}_{3}$ & 22.95 & 12.160 \\
\hline D-(+)-Glucuronic acid $\gamma$-lactone, tris(trimethylsilyl) ether, methyloxime & $\mathrm{C}_{16} \mathrm{H}_{35} \mathrm{NO}_{6} \mathrm{Si}_{3}$ & 23.463 & 9.551 \\
\hline Sedoheptulose, o-methyloxime, hexakis-O-(trimethylsilyl)- & $\mathrm{C}_{26} \mathrm{H}_{65} \mathrm{NO}_{7} \mathrm{Si}_{6}$ & 23.61 & 5.028 \\
\hline d-Glucose, 2,3,4,5,6-pentakis-O-(trimethylsilyl)-, o-methyloxyme, (1Z)- & $\mathrm{C}_{22} \mathrm{H}_{55} \mathrm{NO}_{6} \mathrm{Si}_{5}$ & 23.845 & 0.894 \\
\hline Inositol, 1,2,3,4,5,6-hexakis-O-(trimethylsilyl)-, scyllo- & $\mathrm{C}_{24} \mathrm{H}_{60} \mathrm{O}_{6} \mathrm{Si}_{6}$ & 24.331 & 5.093 \\
\hline D-(-)-Tagatose, pentakis(trimethylsilyl) ether & $\mathrm{C}_{21} \mathrm{H}_{52} \mathrm{O}_{6} \mathrm{Si}_{5}$ & 25.983 & 6.350 \\
\hline Chrysin, bis(trimethylsilyl) ether & $\mathrm{C}_{21} \mathrm{H}_{26} \mathrm{O}_{4} \mathrm{Si}_{2}$ & 30.594 & 2.304 \\
\hline Glucofuranoside, methyl 2,3,5,6-tetrakis-O-(trimethylsilyl)-, a-D- & $\mathrm{C}_{19} \mathrm{H}_{46} \mathrm{O}_{6} \mathrm{Si}_{4}$ & 31.542 & 0.875 \\
\hline D-(+)-Turanose, octakis(trimethylsilyl)ether & $\mathrm{C}_{36} \mathrm{H}_{86} \mathrm{O}_{11} \mathrm{Si}_{8}$ & 31.747 & 7.487 \\
\hline 9,10-Anthracenedione, 2-methyl-1,6-bis[(trimethylsilyl)oxy]- & $\mathrm{C}_{21} \mathrm{H}_{26} \mathrm{O}_{4} \mathrm{Si}_{2}$ & 32.25 & 1.137 \\
\hline D-(-)-Ribofuranose, tetrakis(trimethylsilyl)ether(isomer 1) & $\mathrm{C}_{17} \mathrm{H}_{42} \mathrm{O}_{5} \mathrm{Si}_{4}$ & 32.758 & 8.519 \\
\hline D-(-)-Ribofuranose, tetrakis(trimethylsilyl) ether (isomer 2) & $\mathrm{C}_{17} \mathrm{H}_{42} \mathrm{O}_{5} \mathrm{Si}_{4}$ & 33.889 & 1.727 \\
\hline bis[2-Trimethylsiloxy]ethyl sulfone & $\mathrm{C}_{10} \mathrm{H}_{26} \mathrm{O}_{4} \mathrm{SSi}_{2}$ & 35.507 & 0.301 \\
\hline
\end{tabular}

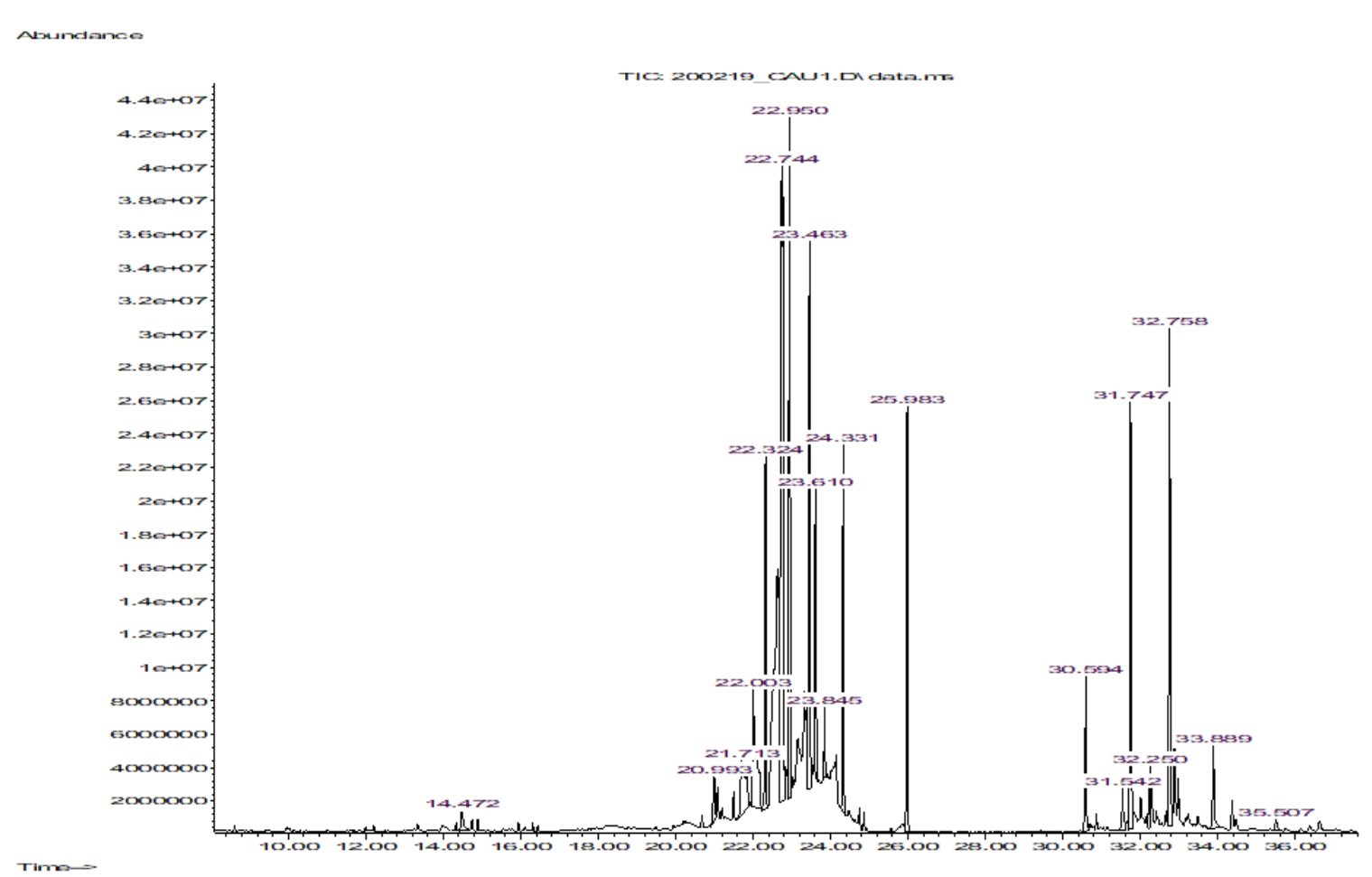

Fig. 4. Chromatogram of ethanol extract of C. auriculata flowers. 
Table 5. Chemical constituents present in ethanol extract of $C$. alata flowers.

\begin{tabular}{|c|c|c|c|}
\hline Name of the Compound & Molecular formula & $\begin{array}{l}\text { Retention } \\
\text { Time (Min) }\end{array}$ & $\begin{array}{l}\text { Total } \\
\text { Percentage }\end{array}$ \\
\hline$\beta$-Citronellol, trifluoroacetate & $\mathrm{C}_{10} \mathrm{H}_{20} \mathrm{O}$ & 14.745 & 0.112 \\
\hline 1,4-Diethoxy-2-nitrobenzene & $\mathrm{C}_{10} \mathrm{H}_{13} \mathrm{NO}_{4}$ & 14.888 & 0.090 \\
\hline 2(3H)-Furanone, dihydro-3,4-bis[(trimethylsilyl)oxy]-, trans- & $\mathrm{C}_{10} \mathrm{H}_{22} \mathrm{O}_{4} \mathrm{Si}_{2}$ & 15.941 & 0.084 \\
\hline 1,5-Anhydro-D-sorbitol, tetrakis(trimethylsilyl) ether & $\mathrm{C}_{18} \mathrm{H}_{44} \mathrm{O}_{5} \mathrm{Si}_{4}$ & 22.007 & 6.379 \\
\hline D-(-)- Erythrofuranose, tris(trimethylsilyl) ether (isomer 2) & $\mathrm{C}_{13} \mathrm{H}_{32} \mathrm{O}_{4} \mathrm{Si}_{3}$ & 22.33 & 6.238 \\
\hline D-Pinitol, pentakis(trimethylsilyl) ether & $\mathrm{C}_{22} \mathrm{H}_{54} \mathrm{O}_{6} \mathrm{Si}_{5}$ & 22.744 & 23.094 \\
\hline ß-D-Mannopyranoside, methyl 2,3,4,6-tetrakis-O-(trimethylsilyl)- & $\mathrm{C}_{19} \mathrm{H}_{46} \mathrm{O}_{6} \mathrm{Si}_{4}$ & 22.954 & 9.539 \\
\hline D-(-)-Tagatose, pentakis(trimethylsilyl) ether, methyloxime (syn) & $\mathrm{C}_{22} \mathrm{H}_{55} \mathrm{NO}_{6} \mathrm{Si}_{5}$ & 23.475 & 13.857 \\
\hline D-(+)-Talose, pentakis(trimethylsilyl) ether, methyloxime (syn) & $\mathrm{C}_{22} \mathrm{H}_{55} \mathrm{NO}_{6} \mathrm{Si}_{5}$ & 23.619 & 9.062 \\
\hline Sedoheptulose, o-methyloxime, hexakis-O-(trimethylsilyl)- & $\mathrm{C}_{26} \mathrm{H}_{65} \mathrm{NO}_{7} \mathrm{Si}_{6}$ & 23.852 & 2.508 \\
\hline Glucopyranose, 2,3-di-O-methyl-1,4,6-tris-O-(trimethylsilyl)- & $\mathrm{C}_{17} \mathrm{H}_{40} \mathrm{O}_{6} \mathrm{Si}_{3}$ & 24.334 & 5.508 \\
\hline Myo-Inositol, 1,2,3,4,5,6-hexakis-O-(trimethylsilyl)- & $\mathrm{C}_{24} \mathrm{H}_{60} \mathrm{O}_{6} \mathrm{Si}_{6}$ & 25.983 & 5.046 \\
\hline Chrysin, bis(trimethylsilyl) ether & $\mathrm{C}_{21} \mathrm{H}_{26} \mathrm{O}_{4} \mathrm{Si}_{2}$ & 30.589 & 0.734 \\
\hline D-(+)-Turanose, octakis(trimethylsilyl) ether & $\mathrm{C}_{36} \mathrm{H}_{86} \mathrm{O}_{11} \mathrm{Si}_{8}$ & 31.755 & 9.725 \\
\hline D-(-)-Ribofuranose, tetrakis(trimethylsilyl) ether (isomer 1) & $\mathrm{C}_{17} \mathrm{H}_{42} \mathrm{O}_{5} \mathrm{Si}_{4}$ & 32.758 & 5.286 \\
\hline 3-a-Mannobiose, octakis(trimethylsilyl) ether (isomer 2) & $\mathrm{C}_{36} \mathrm{H}_{86} \mathrm{O}_{11} \mathrm{Si}_{8}$ & 32.884 & 1.514 \\
\hline D-Ribofuranose, 1,2,3,5-tetrakis-O-(trimethylsilyl)- & $\mathrm{C}_{17} \mathrm{H}_{42} \mathrm{O}_{5} \mathrm{Si}_{4}$ & 33.887 & 0.702 \\
\hline Silane, dimethyl(2-naphthoxy)heptadecyloxy- & $\mathrm{C}_{29} \mathrm{H}_{48} \mathrm{O}_{2} \mathrm{Si}$ & 34.375 & 0.495 \\
\hline
\end{tabular}

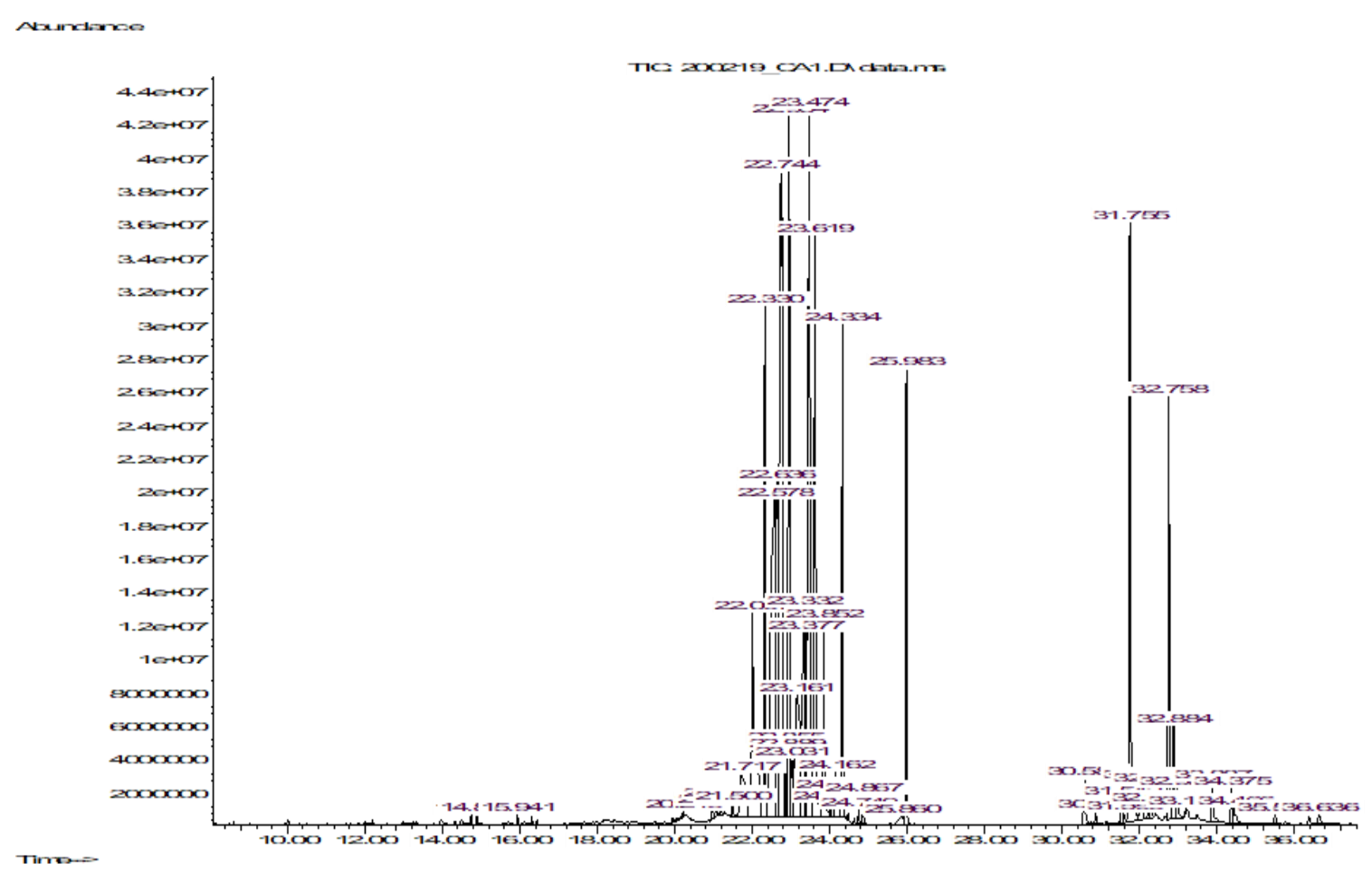

Fig. 5. Chromatogram of ethanol extract of C. alata flowers. 
rubrum was reported by Pereira et al. (2014) and Shin and Lim (2004) indicating its anti-dermatophytic activity. Malassezia is a dermatophytic yeast and the presence of citronellol from ethanolic extract of $C$. alata could have attributed the anti-malassezial activity in this study. Similar work done by Kavitha et al., (2016) reported the susceptibility of Malassezia japonica to citronellol. Chrysin from beewax is reported to protect the skin against pathogenic microorganisms (Gorecka et al., 2020) and in this the study the presence of chry$\sin$ is noted in the ethanolic extract of $C$. alata. Furanones are naturally produced by plants and their antimicrobial activities are widely reported (Sharafutdinov et al., 2019; Rathore et al., 2015; He et al., 2015). The anti-malassezial activity of ethanolic extract of $C$. auriculata and $C$. alata is due to one or other bioactive compounds identified through the GCMS analysis. Further, studies are needed for the purification of the individual compounds and their activity against Malassezia to formulate drugs from Cassia.

Species distribution in the etiology of dandruff is reported (Sharma and Sibi, 2017) and among the Malassezia, different species require different lipids for their growth (Mayser et al., 1997). It is reported that medium-chain triglycerides and medium-chain free fatty acids are toxic for Malassezia species (Papavassilis et al., 1999) and fatty acid monoesters could treat Malassezia associated diseases (Mayser, 2015). Identifying a bioactive compound from plants to control the growth of Malassezia is a way to develop plant-based drugs but different the susceptibility varies between Malassezia species (Sibi et al., 2014).

\section{Conclusion}

Both chloroform and ethanol extracts from C. auriculata and $C$. alata flowers had the ability to inhibit $M$. furfur with MIC values of $50 \mu \mathrm{M}, 88 \mu \mathrm{M}, 75 \mu \mathrm{M}$ and 70 $\mu \mathrm{M}$ thereby could serve as source of potential antidandruff compounds. The presence of fatty acids in chloroform extract and the antifungal compounds from ethanolic extract supported the potential application of Cassia species as potent natural sources for antidandruff activity. Thus, inhibition of $M$. furfur through fatty acids from Cassia flowers is a novel strategy to control dandruff and purification of antidandruff compounds and their mode of inhibition is needed to formulate effective treatment of Malassezia associated diseases.

\section{Conflict of interest}

The authors declare that they have no conflict of interest.

\section{REFERENCES}

1. Ashbee, H. R., Evans, E. G. V. (2002). Immunology of diseases associated with Malassezia species. Clin Microbiol Rev., 15: 21-57. doi: 10.1128/CMR.15.1.21-57.2002
2. Atarzadeh, F., Kamalinejad, M., Dastgheib, L., Amin, G., Jaladat, A.M. and, Nimrouzi, M. (2017). Cassia fistula: A remedy from Traditional Persian Medicine for treatment of cutaneous lesions of Pemphigus vulgaris. Avicenna $J$ Phytomed., 7: 107-115. PMCID PMC5355816

3. Bhattacharyya, A., Jain, N., Prasad, S., Jain, S., Yadav V., Ghosh S., Sengupta, S. (2017). Evaluation of therapeutic potential of VB-001, a leave-on formulation, for the treatment of moderate adherent dandruff. BMC Dermatol., 17: 5. doi: 10.1186/s12895-017-0058-5

4. Cerdeiras MP, Fernandez J, Soubes M, Vero S, Ferreira F, Moyna P, Olano I, Vazquez A (2000). A new antibacterial compound from Ibicella lutea. J Ethnopharmacol., 73: 521-525. doi: 10.1016/S0378-8741(00)00339-1

5. Damodaran, S., Venkataraman, S. (1994). A study on the therapeutic efficacy of Cassia alata, Linn. leaf extract against Pityriasis versicolor. J Ethnopharmacol., 42: 1923. doi: 10.1016/0378-8741(94)90018-3

6. Dilika, F., Bremner, P.D. and, Meyer, J.J.M. (2000). Antibacterial activity of linoleic and oleic acids isolated from Helichrysum pedunculatum: a plant used during circumcision rites. Fitoterapia., 71: 450-452. doi: 10.1016/s0367326x(00)00150-7

7. Duke, J.A. Handbook of Medicinal Herbs. $2^{\text {nd }}$ edition. Florida, Fla, USA: CRC Press; 2002.

8. Findley, K., Oh, J., Yang, J., Conlan, S., Deming, C., Meyer, J. A., Schoenfeld, D., Nomicos, E., Park, M., Kong, H.H. and Segre, J.A. (2013). Topographic diversity of fungal and bacterial communities in human skin. Nature., 498: 367-370. doi: 10.1038/nature12171

9. Gemmer, C.M., Y.M. DeAngelis, Y.M., B. Theelen, B., T. Boekhout, T. and T.L. Dawson Jr, J.L. (2002). Fast, non -invasive method for molecular detection and differentiation of Malassezia yeast species on human skin and application of the method to dandruff microbiology. J Clin Microbiol., 40: 3350-3357. doi: 10.1128/jcm.40.9.33503357.2002

10.Gorecka, A.K., Gorecki, M., Rzepecka-Stojko, A., Balweerz, R. and, Stojko. J. (2020). Bee products in dermatology and skin care. Molecules. 25: 556. doi: $10.3390 /$ molecules25030556

11.Gupta, A.K., Batra, R., Bluhm. R., Boekhout, T. and, Dawson, T.L. (2004). Skin diseases associated with Malassezia species. J. Am. Acad. Dermatol., 51: 785-798. doi: 10.1016/j.jaad.2003.12.034

12.He, X., Du, X., Zang, X., Dong, L., Gu, Z., Cao, L., Chen D., Keyhani NO., Yao, L., Qu, J. and, Guan, X. (2015). Extraction, identification and antimicrobial activity of a new furanone, grifolaone A, from Grifola frondosa. Natural Product Research., 30: 941-947. doi: 10.108 0/14786419.2015.1081197

13.Kavitha, K., Sekar. P., Pirabhakaran, R. and Mukhopadhyay, T. (2016). Incidence of M. japonica in moderate dandruff condition in south India and its sensitivity against tea tree oil, geraniol, citronellol and ketoconazole. International Journal of Recent Scientific Research., 7: 9383-9386.

14.Khare, C.P. (2007). Indian Medicinal Plants: An Illustrated Dictionary. Berlin, Germany: Springer.

15.Kubo, I., Muroi, H. and, Kuo, A. (1993). Antibacterial activity of long-chain alcohols against Streptococcus mutans. J. Agric. Food Chem., 41: 2447-2450. doi: 10.1021/ jf00036a045

16.Kumar, P.P., Kumaravel, S. and, Lalitha, C. (2010). Screening of antioxidant activity, total phenolics and GC- 
MS study of Vitex negundo. Afr. J. Biochem., 4: 191-195. doi: 10.5897/AJBR.9000213

17.Kumar, P.S., Sucheta, S., Deepa, V.S., P. Selvamani, Latha, S. (2008). Evaluation of antidandruff activity using polyherbal oil from six medicinal plants. Journal of Biotechnology., 136: S408-S409. doi: 10.9790/264X0501010106

18.Lee, J.Y., Kim, Y.S. and, Shin, D.H. (2002). Antimicrobial synergistic effect of linolenic acid and monoglyceride against Bacillus cereus and Staphylococcus aureus. J Agric Food Chem., 50: 2193-2199. doi: 10.1021/jf011175a

19.Maity, T.K., Mandal, S.C, Bhakta, T., Pal, M. and, Saha, B.P. (2001). Metabolism of 1,8-dihydroxy 3-hydroxy methyl anthraquinone (aloe-emodin) isolated from the leaves of Cassia tora in albino rats. Phytother Res., 15: 459-460. doi: $10.1002 /$ ptr.975

20.Martindale, W. (1989). The Extra Pharmacopoeia. $28^{\text {th }}$ ed. Pharmaceutical Press; London, UK: pp. 1637-1638.

21.Mayser, P. (2015). Medium chain fatty acid ethyl esters activation of antimicrobial effects by Malassezia enzymes. Mycoses., 58: 215-219. doi: 10.1111/myc.12300

22.Mayser, P., Haze, P., Papavassilis, C., Pickel, M., Gruender, K. and, Gueho, E. (1997). Differentiation of Malassezia species: selectivity of cremophor EL, castor oil and ricinoleic acid for M. furfur. Br J Dermatol., 37: 208213. doi: 10.1046/j.1365-2133.1997.18071890.x.

23.McGraw, L.J., Jager, A.K. and, van Staden, J. (2002). Antibacterial effects of fatty acids and related compounds from plants. South African Journal of Botany., 68: 417423. doi: 10.1016/S0254-6299(15)30367-7

24.Mukherjee, K., Tribedi, P, Mukhopadhyay, B. and, Sil, A.K. (2013). Antibacterial activity of long-chain fatty alcohols against mycobacteria. FEMS Microbiol. Lett., 338: 177-183. doi: 10.1111/1574-6968.12043

25.Murugan, T., Wins, J. A. and, Murugan, M. (2013). Antimicrobial activity and phytochemical constituents of leaf extracts of Cassia auriculata. Ind J Pharm Sci, 75: 122125. doi: $10.4103 / 0250-474 X .113546$

26.Papavassilis, C., Mach, K.K. and, Mayser, P.A. (1999). Medium-chain triglycerides inhibit growth of Malassezia: implications for prevention of systemic infection. Crit. Care Med., 27: 1781-1786. doi: 10.1097/00003246-19990900000013

27.Pereira, F.O., Mendes, J.M., Lima, I.O., Mota, K.S., Oliveira, W.A., Oliveira, W.A. and, Lim, E.O. (2014). Antifungal activity of geraniol and citronellol, two monoterpenes alcohols, against Trichophyton rubrum involves inhibition of ergosterol biosynthesis. Pharm Biol., 53: 228234. doi: $10.3109 / 13880209.2014 .913299$

28.Prabhu, V., Manjula, V., Santhiya, K., Tamileela Kanali, G.P., Saravanakumar, P. and Sibi, G. (2020). Chemometric profile of Calotropis gigantea and its antioxidant activity through bioactive compounds from latex, leaves and flower extracts. Asian Journal of Chemistry., 32: 2865-2872. doi:10.14233/ajchem.2020.22875

29.Rahuman, A.A., Gopalakrishnan, G., Ghouse, B.S., Arumugam, S. and Himalayan, B. (2000). Effect of Feronia limonia on mosquito larvae. Fitoterapia., 71: 553-555. doi: 10.1016/s0367-326x(00)00164-7
30.Rathore, S.S., Isravel, M., Vellaisamy, S., Chellappan, DR., Raman, T. and Ramakrishnan, J. (2017). Exploration of antifungal and immunomodulatory potentials of a furanone derivative to rescue disseminated Cryptococcosis in mice. Sci Rep., 7: 15400. doi: 10.1038/s41598017-15500-8

31.Romagnoli, C., Baldisserotto, A., Vicentini, C.B., Mares, D., Andreotti, E., Vertuani, S. and Manfredini, S. (2016). Antidermatophytic action of resorcinol derivatives: ultrastructural evidence of the activity of phenylethyl resorcinol against Microsporum gypseum. Molecules., 21: 1306. doi: 10.3390/molecules21101306

32.Sampaio, A.L.S.B., Mameri, A.C.A.C.A, Vargas, T.J.D.S., Ramos-eSilva M., Nunes, A.P and, Carneiro, S.C.S.D.S. (2011). Seborrheic dermatitis. An. Bras. Dermatol., 86: 1061-1074. doi: 10.1590/s0365-05962011000600002

33.Sharafutdinov, I.S., Pavlova, A.S., Akhatova, F. S., Khabibrakhmanova, A.M., Rozhina, E.V., Romanova, Y.J., Fakhrullin, R., Lodochnikova, O.A., Kurbangalieva, A. R., Bogachev, M.I. and, Kayumov, A.R. (2019). Unraveling the molecular mechanism of selective antimicrobial activity of 2(5h)-furanone derivative against Staphylococcus aureus. Int J Mol Sci., 20: 694. doi: 10.3390/ijms20030694

34.Sharma, S., and Sibi, G. (2017). Epidemiology and characterization of lipophilic yeast (Malassezia) from human dandruff samples using cultural and molecular methods. International Research Journal of Biochemistry and Biotechnology. 4: 68-74.

35.Shin S. and Lim, S. (2004). Antifungal effects of herbal essential oils alone and in combination with ketoconazole against Trichophyton spp. J Appl Microbiol., 97: 12891296. doi: 10.1111/j.1365-2672.2004.02417.x

36.Sibi, G., Alam, M.A., Shah, J. and Razak, M. (2014). Susceptibility pattern of Malassezia species to selected plant extracts and antifungal agents. International Journal of Green Pharmacy., 8: 226-230. doi: 10.4103/09738258.142675

37.Singhal, M., Kansara, N. (2012). Cassia tora Linn cream inhibits ultraviolet-B-induced psoriasis in rats. ISRN dermatology., 346510. doi: 10.5402/2012/346510

38.Sugita, T., Boekhout, T., Velegraki, A., Guillot, J., Hadina, S. and Cabanes, F.J. (2010). Epidemiology of Malassezia-related skin diseases. T. Boekhout, E. Gueho, P. Mayser, A. Velegraki (Eds.,), Malassezia and the skin, Springer Verlag. pp. 65-119. doi: 10.1007/978-3-642-036 16-3_3

39.Takemoto, A., Cho, O., Morohoshi, Y., Sugita, T. and, Muto, M. (2015). Molecular characterization of the skin fungal microbiome in patients with psoriasis. $J$ Dermatol., 42: 166-170. doi: 10.1111/1346-8138.12739

40.Timothy, S.Y., Wazis, C.H., Adati, R.G. and Maspalma, I.D. (2012). Antifungal activity of aqueous and ethanolic leaf extracts of Cassia alata Linn. Journal of Applied Pharmaceutical Science., 2: 182-185. doi: 10.7324/ JAPS.2012.2728

41.Togashi, N., Shiraishi, A., Nishizaka, M., Matsuoka, K., Endo, K., Hamashima., H and Inoue, Y. (2007). Antibacterial activity of long-chain fatty alcohols against Staphylococcus aureus. Molecules., 12: 139-148. doi: 10.3390/12 020139 